\title{
H.E.S.S. observations of PKS 2155-304
}

F. Aharonian ${ }^{1}$, A. G. Akhperjanian ${ }^{2}$, K.-M. Aye ${ }^{3}$, A. R. Bazer-Bachi ${ }^{4}$, M. Beilicke ${ }^{5}$, W. Benbow ${ }^{1}$, D. Berge ${ }^{1}$, P. Berghaus ${ }^{6, \star}$, K. Bernlöhr ${ }^{1,7}$, O. Bolz ${ }^{1}$, C. Boisson ${ }^{8}$, C. Borgmeier ${ }^{7}$, F. Breitling ${ }^{7}$, A. M. Brown ${ }^{3}$, J. Bussons Gordo $^{9}$, P. M. Chadwick ${ }^{3}$, V. R. Chitnis ${ }^{10,20, \star \star}$, L.-M. Chounet ${ }^{11}$, R. Cornils ${ }^{5}$, L. Costamante ${ }^{1,20}$, B. Degrange ${ }^{11}$, A. Djannati-Atai ${ }^{6}$, L.O'C. Drury ${ }^{12}$, T. Ergin $^{7}$, P. Espigat ${ }^{6}$, F. Feinstein ${ }^{9}$, P. Fleury ${ }^{11}$, G. Fontaine ${ }^{11}$, S. Funk ${ }^{1}$, Y. A. Gallant ${ }^{9}$, B. Giebels ${ }^{11}$, S. Gillessen ${ }^{1}$, P. Goret ${ }^{13}$, J. Guy ${ }^{10}$, C. Hadjichristidis ${ }^{3}$, M. Hauser ${ }^{14}$, G. Heinzelmann ${ }^{5}$, G. Henri ${ }^{15}$, G. Hermann ${ }^{1}$, J. A. Hinton ${ }^{1}$, W. Hofmann ${ }^{1}$, M. Holleran ${ }^{16}$, D. Horns ${ }^{1}$, O. C. de Jager ${ }^{16}$, I. Jung ${ }^{1,14, \star \star \star}$, B. Khélifi ${ }^{1}$, Nu. Komin ${ }^{7}$, A. Konopelko ${ }^{1,7}$, I. J. Latham ${ }^{3}$, R. Le Gallou ${ }^{3}$, M. Lemoine ${ }^{11}$, A. Lemière ${ }^{6}$, N. Leroy ${ }^{11}$, T. Lohse ${ }^{7}$, A. Marcowith ${ }^{4}$, C. Masterson ${ }^{1,20}$, T. J. L. McComb ${ }^{3}$, M. de Naurois ${ }^{10}$, S. J. Nolan ${ }^{3}$, A. Noutsos ${ }^{3}$, K. J. Orford ${ }^{3}$, J. L. Osborne ${ }^{3}$, M. Ouchrif ${ }^{10,20}$, M. Panter ${ }^{1}$, G. Pelletier ${ }^{15}$, S. Pita ${ }^{6}$, M. Pohl ${ }^{17, \dagger}$, G. Pühlhofer ${ }^{1,14}$,

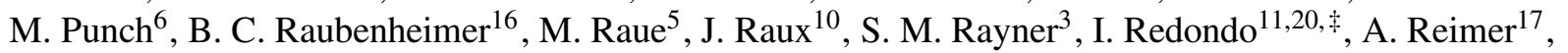
O. Reimer ${ }^{17}$, J. Ripken ${ }^{5}$, M. Rivoal ${ }^{10}$, L. Rob ${ }^{18}$, L. Rolland ${ }^{10}$, G. Rowell ${ }^{1}$, V. Sahakian ${ }^{2}$, L. Saugé ${ }^{15}$, S. Schlenker ${ }^{7}$, R. Schlickeiser ${ }^{17}$, C. Schuster ${ }^{17}$, U. Schwanke ${ }^{7}$, M. Siewert ${ }^{17}$, H. Sol ${ }^{8}$, R. Steenkamp ${ }^{19}$, C. Stegmann ${ }^{7}$, J.-P. Tavernet ${ }^{10}$, C. G. Théoret ${ }^{6}$, M. Tluczykont ${ }^{11,20}$, D. J. van der Walt ${ }^{16}$, G. Vasileiadis ${ }^{9}$, P. Vincent ${ }^{10}$, B. Visser ${ }^{16}$, H. J. Völk ${ }^{1}$, and S. J. Wagner ${ }^{14}$

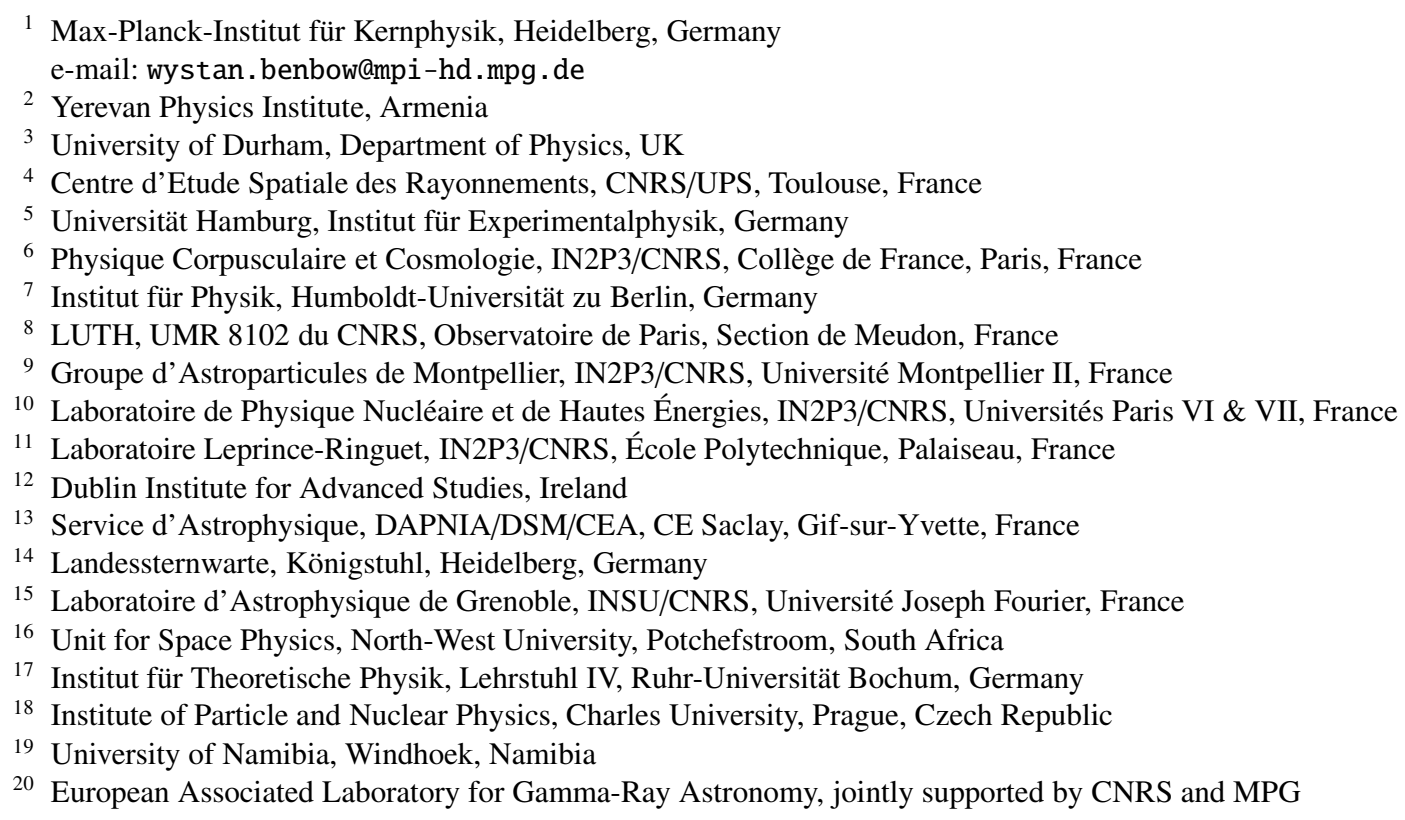

Received 17 August 2004 / Accepted 27 September 2004

\footnotetext{
* Université Libre de Bruxelles, Faculté des Sciences, Belgium.

$\star \star$ Now at Tata Institute of Fundamental Research, Mumbai, India.

$\star \star \star$ Now at Washington Univ., Department of Physics, St. Louis, MO, USA.

Now at Department of Physics and Astronomy, Iowa State University, Ames, Iowa, USA.

Now at Department of Physics and Astronomy, Univ. of Sheffield, UK.
} 


\begin{abstract}
The high-frequency peaked BL Lac PKS 2155-304 at redshift $z=0.117$ has been detected with high significance $(\sim 45 \sigma)$ at energies greater than $160 \mathrm{GeV}$, using the H.E.S.S. stereoscopic array of imaging air-Cherenkov telescopes in Namibia. A strong signal is found in each of the data sets corresponding to the dark periods of July and October, 2002, and June-September, 2003. The observed flux of VHE gamma rays shows variability on time scales of months, days, and hours. The monthly-averaged integral flux above $300 \mathrm{GeV}$ varies between $10 \%$ and $60 \%$ of the flux observed from the Crab Nebula. Energy spectra are measured for these individual periods of data taking and are characterized by a steep power law with a time-averaged photon index of $\Gamma=3.32 \pm 0.06$. An improved $\chi^{2}$ per degree of freedom is found when either a power law with an exponential cutoff energy or a broken power law are fit to the time-averaged energy spectrum. However, the significance of the improvement is marginal $(\sim 2 \sigma)$. The suggested presence of features in the energy spectrum may be intrinsic to the emission from the blazar, or an indication of absorption of $\mathrm{TeV}$ gamma rays by the extragalactic infrared background light.
\end{abstract}

Key words. galaxies: active - galaxies: BL Lacertae objects: individual: PKS 2155-304 - gamma rays: observations

\section{Introduction}

The high-frequency peaked BL Lac (HBL) object PKS 2155-304 $(z=0.117)$ was discovered by the HEAO 1 X-ray satellite (Griffiths et al. 1979; Schwartz et al. 1979) at a position consistent with a poorly localized detection by the Ariel $V$ satellite (Cooke et al. 1978). PKS 2155-304 is now known to be one of the brightest extragalactic $\mathrm{X}$-ray sources in the sky and as a result other X-ray satellites, including ROSAT, BeppoSAX, RXTE, and Chandra (see, e.g., Brinkmann et al. 1994; Chiappetti \& Torroni 1997; Vestrand \& Sreekumar 1999; Nicastro et al. 2002), have detected it on a regular basis. PKS 2155-304 is well studied and has a history of strong broad-band variability. It is associated with a compact, flat-spectrum radio source, and exhibits an essentially featureless continuum from radio to $\mathrm{X}$-ray frequencies. The maximum power emitted by PKS 2155-304 is between the UV and soft X-ray range, and it is the brightest BL Lac detected in the UV regime (Wandel \& Urry 1991). Gamma-ray emission in the energy range $30 \mathrm{MeV}$ to $10 \mathrm{GeV}$ was detected from PKS 2155-304 by the EGRET detector aboard the Compton Gamma Ray Observatory satellite (Vestrand et al. 1995). The EGRET observations indicated a hard energy spectrum with integral power-law photon index of $1.71 \pm 0.24$. All of these characteristics make PKS 2155-304 a likely emitter of $>100 \mathrm{GeV}$ gamma rays. In fact, it was detected at energies greater than $300 \mathrm{GeV}$ in 1996 and 1997 by the University of Durham Mark 6 Telescope as reported in Chadwick et al. (1999a). However, the object was not detected by the same instrument in 1998 (Chadwick et al. 1999b). Furthermore, PKS 2155-304 was not detected by the CANGAROO experiment during observations made in 1997 (Roberts et al. 1999), 1999 (Nishijima et al. 2001), 2000 or 2001 (Nakase et al. 2003). The reported upper limit from CANGAROO in 1997 is consistent with the flux detected by Durham, and the lack of a detection of PKS 2155-304 at TeV energies in subsequent years is not inconsistent with the fact that such emission from AGN is known to be highly variable.

The present confirmation of the detection of VHE gammarays from PKS 2155-304 by the High Energy Stereoscopic System (H.E.S.S.) along with observations at other wavelengths will yield considerable insight into the mechanisms for $\mathrm{TeV}$ gamma-ray emission from blazars, help delineate the differences between low-frequency peaked BL Lacs and HBLs, and will assist in the understanding of the extragalactic infrared background light (EBL). This latter point is strengthened by the fact that PKS 2155-304 is the second most distant object detected at $\mathrm{TeV}$ energies and therefore its energy spectrum may exhibit characteristics, such as steepening of the spectrum and a cutoff, caused by absorption of VHE gamma rays via pair production on the EBL. The most distant object detected at TeV energies, 1ES $1426+428(z=0.129)$, showed features in its energy spectrum which have been interpreted as consequences of such absorption (Aharonian et al. 2002a). In addition, variability studies using detailed light curves obtained from observations of PKS 2155-304 can place strong constraints on the physical modeling of blazars.

\section{H.E.S.S. detector}

The H.E.S.S. experiment has been operating since June, 2002 , in the Khomas Highlands of Namibia $\left(23^{\circ} 16^{\prime} 18^{\prime \prime} \mathrm{S}\right.$, $16^{\circ} 30^{\prime} 1^{\prime \prime} \mathrm{E}, 1835 \mathrm{~m}$ above sea level). The detector consists of a system of four imaging air-Cherenkov telescopes in a square of $120 \mathrm{~m}$ side. Each individual telescope is an alt-az mount Davies-Cotton reflector (Davies \& Cotton 1957) with a flat-toflat width of $13 \mathrm{~m}$, and has a camera mounted at the focal length of $15 \mathrm{~m}$. The total mirror area of each telescope is $107 \mathrm{~m}^{2}$, segmented into 382 individual round $(60 \mathrm{~cm}$ diameter) frontaluminized glass mirrors. The H.E.S.S. cameras provide a $5^{\circ}$ field of view and contain 960 individual photomultiplier (PMT) pixels subtending $0.16^{\circ}$ each, with Winston cone light concentrators. The camera is modular in design, housing 60 drawers of 16 PMTs each, and contains all the necessary electronics for operation, triggering, and readout. The trigger electronics divide the camera into overlapping 64 PMT sectors (4 adjacent drawers) with a trigger requirement that a sector has a minimum number of pixels with a signal above a threshold in photoelectrons (PEs) coincident in an effective $\sim 1.3 \mathrm{~ns}$ trigger window. Upon receiving a camera trigger, the signal stored in analog memories from each of the PMTs, sampled at $1 \mathrm{GHz}$, is integrated within a $16 \mathrm{~ns}$ window. Once a camera has triggered, a signal is sent out via an optical fiber to a central trigger system (Funk et al. 2004) which allows for a multiple telescope coincidence requirement. More details on H.E.S.S. can be found in Bernlöhr et al. (2003); Hofmann (2003); Vincent et al. (2003).

\section{Observations}

The H.E.S.S. observations of PKS 2155-304 were made while the system was under construction, therefore the data cover 
Table 1. Shown are the dark periods in which PKS 2155-304 was observed, the configuration of H.E.S.S. during those observations, the telescope multiplicity required to trigger the array, the number of telescopes available, the sector trigger requirement (number of pixels above a threshold in PEs), the observation mode, the number of on-source runs, the dead time corrected observation time, the system rate, the dead time percentage, and the mean zenith angle of the observations $\left(Z_{\mathrm{obs}}\right)$.

\begin{tabular}{cccccccccc}
\hline \hline $\begin{array}{c}\text { Dark } \\
\text { period }\end{array}$ & Configuration & $\begin{array}{c}N_{\text {tel }} \\
\text { Mult., Tot. }\end{array}$ & $\begin{array}{c}\text { Sector } \\
\text { trigger }\end{array}$ & $\begin{array}{c}\text { Obs. } \\
\text { mode }\end{array}$ & $\begin{array}{c}\text { No. } \\
\text { Runs }\end{array}$ & $\begin{array}{c}\text { Obs. time } \\
{[\mathrm{h} \mathrm{live}]}\end{array}$ & $\begin{array}{c}\text { Syst. rate } \\
{[\mathrm{Hz}]}\end{array}$ & $\begin{array}{c}\text { Dead time } \\
\%\end{array}$ & $\begin{array}{c}Z_{\text {obs }} \\
{\left[{ }^{\circ}\right]}\end{array}$ \\
\hline $07 / 2002$ & Mono (30 drawers) & 1,1 & $4>6.7$ & On-Off & 8 & 2.5 & 130 & 24 & 15 \\
$10 / 2002$ & Mono (52 drawers) & 1,1 & $4>6.7$ & On-Off & 16 & 4.3 & 230 & 37 & 16 \\
$11 / 2002$ & Mono & 1,1 & $4>6.7$ & On-Off & 4 & 0.8 & 250 & 53 & 35 \\
$06 / 2003$ & Software stereo & 2,2 & $4>6.7$ & Wobble & 32 & 10.8 & 60 & 23 & 13 \\
$07 / 2003$ & Hardware stereo & 2,2 & $3>5.3$ & Wobble & 55 & 22.1 & 100 & 7 & 24 \\
$08 / 2003$ & Hardware stereo & 2,2 & $3>5.3$ & Wobble & 45 & 19.6 & 100 & 7 & 14 \\
$09 / 2003$ & Hardware stereo & 2,2 & $3>5.3$ & Wobble & 2 & 0.9 & 120 & 8 & 8 \\
$09 / 2003$ & Hardware stereo & 2,3 & $3>5.3$ & Wobble & 6 & 2.1 & 170 & 12 & 18 \\
\hline
\end{tabular}

various telescope configurations and trigger criteria. Observations in 2002 were made with just one telescope. In January 2003, a second telescope was added to the array and observations in 2003 consist mostly of a two-telescope configuration. The exception to this is in September 2003, when a third telescope was added to the array. The fourth and final telescope was added to the array in December 2003, after the end of the observation season for PKS 2155-304.

The trigger for H.E.S.S. has similarly evolved. During the observations in July, October, November 2002, and June 2003, the sector trigger consisted of a requirement for 4 pixels each with more than 6.7 PEs. However, the July and October 2002 observations were made with only the inner 30 and 52 drawers, respectively, participating in the trigger while the entire camera was used for image collection. For all later observations the cameras were used fully. The system level trigger was installed in July 2003. Before this time two-telescope data were taken with each telescope separately, and the stereo multiplicity requirement (two telescopes) was performed off-line ("Software Stereo") using GPS time stamps. After the installation of the central trigger system the stereo multiplicity requirement was performed in the hardware ("Hardware Stereo"). As the system rate was considerably lower due to a two-telescope multiplicity requirement, the sector trigger requirement could be lowered to 3 pixels with more than 5.3 PEs, allowing for a lower energy threshold. This increased the individual telescope rates, from $\sim 250 \mathrm{~Hz}$ to $\sim 800 \mathrm{~Hz}$, while maintaining a reduced system dead time.

Table 1 gives details of the observations of PKS 2155-304 by H.E.S.S. which pass conservative run selection criteria. These run selection criteria remove runs for which the sky was not clear and where the telescopes were not operating within specified requirements (e.g., trigger rate stability, more than $95 \%$ of the camera pixels operational). All the single telescope observation runs were taken in $O n-O f f$ mode. Here, data were taken on source for $25 \mathrm{~min}$, preceded or followed by offsource observations, used to estimate the background. These off-source observations differ by $\pm 30 \mathrm{~min}$ in right ascension (RA) from the actual source position. Given an approximately $5 \mathrm{~min}$ (reduced to $2 \mathrm{~min}$ after 2002) transition time for the data acquisition system, this ensures that the on and off runs have identical azimuth and altitude profiles. The stereo data were taken in 28 min runs using Wobble mode. In this mode, the source direction is positioned $\pm 0.5^{\circ}$ in declination relative to the center of the field of view of the camera during observations. The sign of the offset is alternated in successive scans to reduce systematic effects. Due to the large field of view of the H.E.S.S. cameras, use of Wobble mode allows for both onsource observations and simultaneous estimation of the background induced by charged cosmic rays, since the background can be estimated from different regions in the same field of view. This eliminates the need for off-source observations and therefore doubles the amount of time available for on-source observations.

\section{Technique}

\subsection{Event reconstruction}

The analysis of the data passing the run selection criteria proceeds in the following steps: first the images are calibrated (Aharonian et al. 2004) and then "cleaned" to remove noise from the image. The image cleaning is done using a twostage tail-cut procedure which requires a pixel to have a signal greater than 10 PEs and a neighboring pixel to have a signal larger than 5 PEs. Also pixels greater than 5 PEs are included if they have a neighbor greater than 10 PEs. After this image cleaning is performed, the moments of the shower image are parameterized using a Hillas-type analysis (Hillas 1985). The shower geometry is reconstructed using stereoscopic techniques (Aharonian et al. 1999b) with a typical angular resolution of $\sim 0.1^{\circ}$ and an average accuracy of $\sim 10 \mathrm{~m}$ in the determination of the shower core location. To ensure that the analyzed images are not truncated by the edge of the camera, only images which pass a distance cut requiring the image center of gravity to be less than $2^{\circ}$ from the center of the camera are used in the reconstruction. For the stereo configuration a minimum of 2 telescopes meeting this criterion are required for a successful reconstruction. In addition, at least two telescopes are each required to exceed a minimum total signal to ensure that the images are well reconstructed. The analysis techniques are similar for the mono-configurations, although a necessary difference arises in the determination of the shower geometry. 
Table 2. The selection cuts applied to the data and the percentage of $\gamma$-ray and background events retained by those cuts.

\begin{tabular}{lcccccccccccc}
\hline \hline Configuration & $N_{\text {tel }}$ & $\begin{array}{c}\text { MRSL } \\
\min \\
{[\sigma]}\end{array}$ & $\begin{array}{c}\text { MRSL } \\
\max \end{array}$ & $\begin{array}{c}\text { MRSW } \\
\min \\
{[\sigma]}\end{array}$ & $\begin{array}{c}\text { MRSW } \\
\max \\
{[\sigma]}\end{array}$ & $\begin{array}{c}\text { C.O.G. } \\
\max \\
{[\mathrm{mrad}]}\end{array}$ & $\begin{array}{c}\text { Size } \\
\min \end{array}$ & $\begin{array}{c}\text { Length/Size } \\
\max \end{array}$ & $\begin{array}{c}N_{\text {tel }} \\
\min \end{array}$ & $\begin{array}{c}\theta^{2} \\
\max / \mathrm{PE}]\end{array}$ & $\begin{array}{c}\gamma \\
{\left[\begin{array}{c}\left.\mathrm{max}^{2}\right] \\
\left.\mathrm{deg}^{2}\right]\end{array}\right.}\end{array}$ & $\begin{array}{c}\text { BG } \\
\%\end{array}$ \\
\hline Mono & 1 & -5.0 & 3.4 & -4.2 & 1.0 & 35 & None & 0.016 & 1 & 0.035 & 20 & 0.012 \\
Stereo (Soft) & 2 & -2.2 & 3.2 & -10.0 & 1.6 & 35 & 75 & None & 2 & 0.030 & 72 & 0.036 \\
Stereo (Hard) & 2 & -1.8 & 2.0 & -10.0 & 1.0 & 35 & 55 & None & 2 & 0.025 & 48 & 0.029 \\
Stereo (Hard) & 3 & -1.8 & 2.0 & -10.0 & 1.0 & 35 & 55 & None & 2 & 0.025 & 54 & 0.041 \\
\hline
\end{tabular}

In the mono-telescope case, the impact parameter and direction of the shower axis are estimated directly from the image. To do this, the angular distance, along the major axis of the reconstructed ellipse, from the image center of gravity to the point of origin of the event is estimated using the image parameter construction length/log (size) (Masterson 2000).

\subsection{Background rejection}

After the event reconstruction, the much more numerous cosmic-ray background events are rejected using cuts on mean reduced scaled width (MRSW) and length (MRSL) parameters. These parameters are defined as the mean of the difference in standard deviations for each telescope of the width (length) observed in the image from that which is expected from gammaray simulations ( $\langle$ width $\rangle$ and $\sigma$ ) based on image intensity, reconstructed impact parameter and zenith angle of observations. The equation for MRSW is as follows:

$$
M R S W=\frac{1}{N_{\text {tel }}} \sum_{i=0}^{N_{\text {tel }}} \frac{\text { width }_{i}-\langle\text { width }\rangle_{i}}{\sigma_{i}},
$$

and is similar for MRSL. Images from muons are rejected by requiring events be coincident in at least 2 telescopes in the stereo configuration or by using a cut on length divided by size of the image, after image cleaning, for the mono-configuration (Catanese et al. 1995). A cut on $\theta^{2}$, the square of the angular difference between the reconstructed shower position and the source position, is applied and is equivalent to placing the data into a round bin centered on the source position. All the cuts (shown in Table 2) are optimized a priori (simultaneously) to yield the maximum expected significance per hour of observation. The data used for optimization consists of Monte Carlo gamma-ray simulations at a zenith angle of $20^{\circ}$ with a Crablike energy spectrum (spectral index $=2.6,1 / 2 \mathrm{Crab}$ flux) and real off-source data. The percentage of gamma-rays and cosmic rays retained by the cuts is also shown in Table 2 . The significance expected (and observed) is not strongly dependent on the exact values of the cuts used. For each of the various hardware configurations a different set of cuts is used, all optimized on Monte Carlo simulations and comparable off-source data sets.

\subsection{Background estimation}

For mono-telescope observations the background is estimated from the number of events passing the selection cuts at the center of the field of view during off-source observations. The background is then normalized using the ratio of dead time corrected observation time. For the stereo (Wobble) observations
Table 3. Shown are the number of on-source and off-source events passing the cuts, the normalization for the off-source events, the observed excess from PKS 2155-304, and the significance of the excess for each of the dark periods.

\begin{tabular}{ccccccc}
\hline \hline $\begin{array}{c}\text { Dark } \\
\text { Period }\end{array}$ & $N_{\text {tels }}$ & On & Off & Norm & Excess & $\begin{array}{c}\text { Sig. } \\
{[\sigma]}\end{array}$ \\
\hline $07 / 2002$ & 1 & 637 & 234 & 1.063 & 388 & 13.0 \\
$10 / 2002$ & 1 & 865 & 519 & 1.065 & 312 & 8.2 \\
$11 / 2002$ & 1 & 80 & 90 & 0.896 & -0.65 & -0.1 \\
$06 / 2003$ & 2 & 1396 & 4619 & 0.152 & 694 & 21.1 \\
$07 / 2003$ & 2 & 3169 & 13643 & 0.147 & 1164 & 22.1 \\
$08 / 2003$ & 2 & 3369 & 12955 & 0.147 & 1459 & 27.7 \\
$09 / 2003$ & 2 & 185 & 697 & 0.148 & 82 & 6.7 \\
$09 / 2003$ & 3 & 1005 & 3946 & 0.147 & 425 & 14.7 \\
\hline Total & Stereo & & & & & 43.8 \\
Total & All & & & & & 44.9 \\
\hline
\end{tabular}

the background is estimated using all events passing cuts in a ring around the source location. The distance from the source position to the radial center of the ring is $0.5^{\circ}$, and the width of the ring is adjusted such that the area of the ring is approximately seven times the area of the on-source region. The normalization of the off-source event total is corrected for the radial acceptance of the camera. The use of a larger background region in this ring technique reduces the relative statistical error on the background measurement. After the number of onsource and background events passing the selection cuts are determined, the significance of the excess is calculated following the method of Eq. (17) in Li \& Ma (1983).

\section{Detection of PKS 2155-304}

PKS 2155-304 is strongly detected in all the dark periods in which it was observed with the exception of November 2002 where the exposure was less than one hour. Table 3 shows the results of the H.E.S.S. observations for each of the individual dark periods. The total significance of the excess for all observations is $44.9 \sigma$. Figure 1 shows the on-source and normalized off-source distributions of $\theta^{2}$ for all observations in the twotelescope hardware stereo configuration ${ }^{1}$. The background is flat in $\theta^{2}$ as expected, and there is a clear excess at small values of $\theta^{2}$ corresponding to the observed signal. Figure 2 shows a

1 This configuration represents the major share of the data; the shapes of the respective curves are similar for the other configurations. 


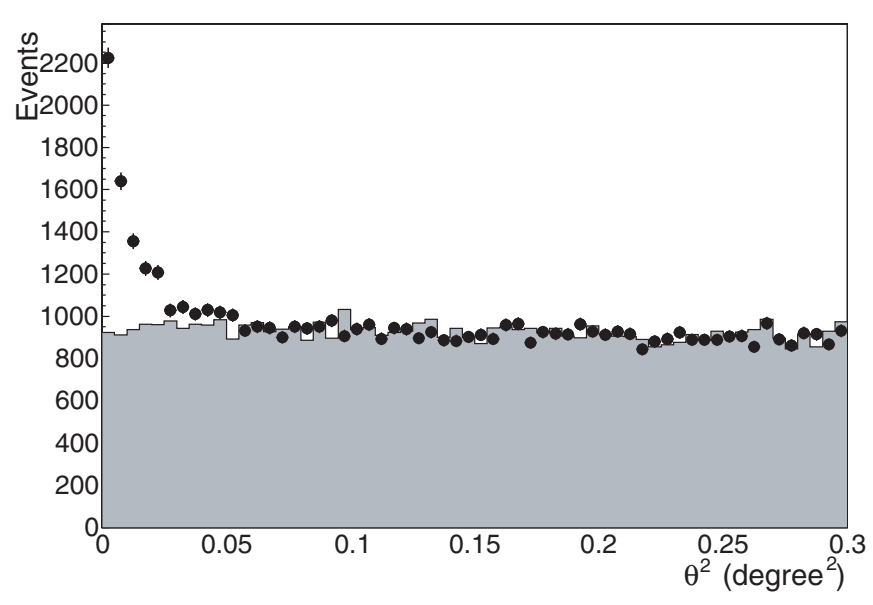

Fig. 1. The distribution of $\theta^{2}$ for on-source events (points) and normalized off-source events (shaded) from observations of PKS 2155-304 in the two-telescope hardware stereo configuration. For this plot, the background is taken from a region of comparable area located at the opposite offset position in the field of view. This causes the significance shown to be reduced due to the smaller area used for the background estimation.

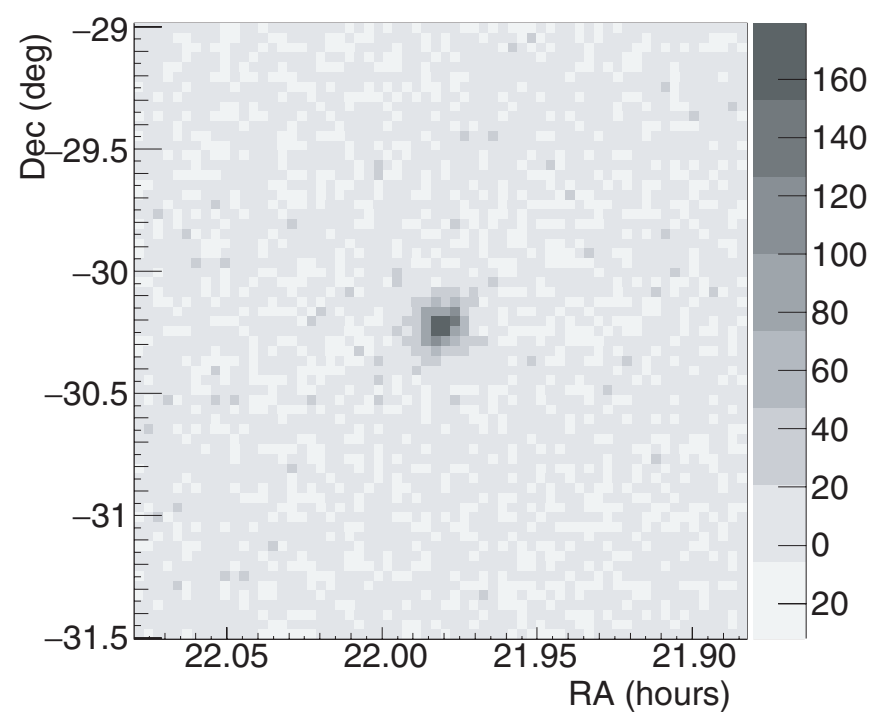

Fig. 2. A two dimensional distribution of excess events observed in the direction of PKS 2155-304 for the two-telescope hardware stereo configuration.

two-dimensional sky map of the excess observed in the direction of PKS 2155-304 for the same configuration. The bins are not correlated and represent the actual distribution of observed gamma-rays (and some background events) on the sky. A fit of the peak in Fig. 2 to a two-dimensional Gaussian finds the shape of the excess to be characteristic of a point source. The fit peak is located $\left(\Delta_{\mathrm{RA}}=9 \pm 12_{\text {stat }} \operatorname{arcsec}, \Delta_{\mathrm{dec}}=14 \pm 10_{\text {stat }} \operatorname{arcsec}\right)$ from the position of PKS 2155-304, consistent with the position of the blazar as expected (systematic errors on the pointing are less than 20 arcsec in RA and declination (Gillessen 2003)).

Figure 3 shows the distributions of MRSW, MRSL, and $\theta^{2}$ for the observed excess after all cuts are applied (excluding both the MRSW and MRSL cuts in (a) and (b), and the $\theta^{2}$ cut in (c)), as well as the corresponding distributions for Monte Carlo gamma-ray simulations, for the entire twotelescope hardware stereo data set. The agreement is good and shows that the simulations accurately characterize the detector response and that the detector performs as expected. In particular, the agreement of the $\theta^{2}$ distribution demonstrates that the point spread function (width $<0.1^{\circ}$ ) is well understood.

\section{Energy spectrum}

\subsection{Technique}

The energy of observed gamma rays, $E_{\text {fit }}$, is calculated using the mean of the energies estimated for each telescope with a typical event resolution of $\sim 15 \%$. Each of the individual energy estimates use the image size, impact parameter ${ }^{2}$ of the event, and the zenith angle of observation $(Z)$ for each telescope. The energy estimates are based on results from Monte Carlo gamma-ray simulations. For the determination of the energy spectrum the data are logarithmically binned ( 8 bins per decade) in energy. However, only bins for which the average bias in $E_{\text {fit }}$ is less than $10 \%$ are used, effectively placing a lower energy threshold on suitable events. This is done to eliminate any systematic effects that might arise because energy estimates for events near the trigger threshold yield, on average, too large an energy. The range of bins used varies for each individual run due to the zenith angle of observation and results in the bins at lower energies having differing amounts of exposure. For each event passing cuts the effective area, $A_{\text {eff }}\left(E_{\text {fit }}, Z\right)$, is individually determined. The influence of the finite energy resolution has been taken into account in the effective areas which are fit as a function of $Z$ and $E_{\text {fit }}$ for each of the various observation configurations similar to the method in Mohanty et al. (1998). For each bin, the sum of $1 / A_{\text {eff }}$, is calculated for both on-source $\left(O_{i}\right)$ and off-source $\left(B_{i}\right)$ events using only events from the appropriate runs. Each bin in the spectrum then contains:

$F_{i}=\frac{\left(O_{i}-B_{i}\right)}{\Delta E_{i} t_{i}}$,

where $t_{i}$ and $\Delta E_{i}$ are the exposure time and width in energy respectively for that bin. As the collection area depends weakly on the spectral shape assumed, an iterative procedure is followed, where the initial spectral shape is assumed to be flat in $v F_{v}$ (differential power-law index $=2.0$ ). When deviations from this hypothesis are found the collection area is recalculated using the previous result and the spectrum is determined again. This procedure continues until convergence (typically 1-2 iterations).

\subsection{Dark period spectra}

Using the observed excess of gamma rays, a differential spectrum for each darkness period during which PKS 2155-304 was detected is generated, and is shown in Fig. 4. These spectra are fit to a power law:

$\frac{\mathrm{d} N}{\mathrm{~d} E}=I_{0}\left(\frac{E}{E_{0}}\right)^{-\Gamma}$

2 The distance of the center of gravity of the image from the center of the field of view is used in the mono-telescope configuration. 


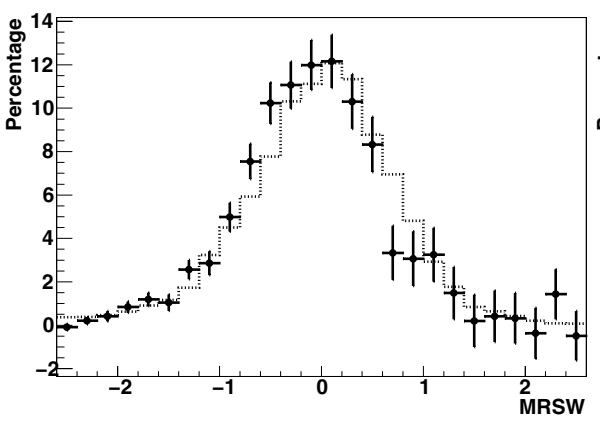

(a)

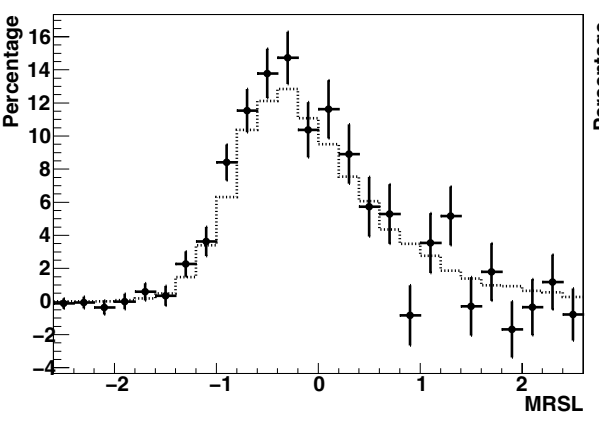

(b)

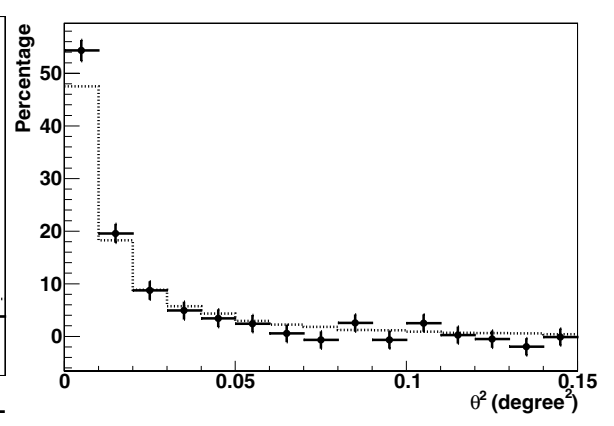

(c)

Fig. 3. The distributions of a) mean reduced scaled width (MRSW), b) mean reduced scaled length (MRSL), and c) $\theta^{2}$ for the background subtracted signal after selection cuts (excluding both the MRSW and MRSL cuts in a) and b), and the $\theta^{2}$ cut in c)) from observations of PKS 2155-304 (points) and similarly for Monte Carlo gamma-ray simulations (spectral index $=3.35$, zenith angle $=20^{\circ}$ ) in the two-telescope hardware stereo configuration (line).

Table 4. The results of power law fits to the differential energy spectrum of PKS 2155-304 for each dark period. Shown are $I_{0}$, $\Gamma$, the $\chi^{2}$ and degrees of freedom (d.o.f.) for the fit, the integral flux above $1 \mathrm{TeV}(I(>1 \mathrm{TeV})), I(>300 \mathrm{GeV}), I\left(>E_{\mathrm{th}}\right)$ where $E_{\text {th }}$ is the post-selection cuts energy threshold of the observations, and the percentage of these fluxes relative to the respective values measured by H.E.S.S. from the Crab Nebula. Only the statistical errors are shown.

\begin{tabular}{|c|c|c|c|c|c|c|c|c|c|c|}
\hline $\begin{array}{r}\text { Dark } \\
\text { period }\end{array}$ & {$\left[\begin{array}{c}I_{0} \\
\frac{10^{-8}}{\mathrm{~m}^{2} \mathrm{~s} \mathrm{TeV}}\end{array}\right]$} & $\Gamma$ & $\chi^{2}$ (d.o.f.) & $\begin{array}{c}I(>1 \mathrm{TeV}) \\
{\left[\frac{10^{-9}}{\mathrm{~m}^{2} \mathrm{~s}}\right]}\end{array}$ & $\begin{array}{c}\text { Crab } \\
\%\end{array}$ & $\begin{array}{c}I(>300 \mathrm{GeV}) \\
{\left[\frac{10^{-7}}{\mathrm{~m}^{2} \mathrm{~s}}\right]}\end{array}$ & $\begin{array}{c}\text { Crab } \\
\%\end{array}$ & $\begin{array}{c}E_{\mathrm{th}} \\
{[\mathrm{GeV}]}\end{array}$ & $\begin{array}{l}I\left(>E_{\mathrm{th}}\right) \\
{\left[\frac{10^{-7}}{\mathrm{~m}^{2} \mathrm{~s}}\right]}\end{array}$ & $\begin{array}{c}\text { Crab } \\
\%\end{array}$ \\
\hline $07 / 2002$ & $15.6 \pm 2.1$ & $2.84 \pm 0.24$ & $7.0(7)$ & $85.1 \pm 16.1$ & 43 & $7.77 \pm 1.63$ & 56 & 300 & $7.77 \pm 1.63$ & 56 \\
\hline $10 / 2002$ & $6.36 \pm 1.75$ & $3.10 \pm 0.46$ & $0.5(4)$ & $30.4 \pm 10.6$ & 15 & $3.79 \pm 1.63$ & 28 & 300 & $3.79 \pm 1.63$ & 28 \\
\hline $06 / 2003$ & $2.42 \pm 0.28$ & $3.56 \pm 0.17$ & $4.0(5)$ & $9.45 \pm 1.27$ & 4.8 & $2.06 \pm 0.37$ & 15 & 260 & $2.97 \pm 0.59$ & 17 \\
\hline $07 / 2003$ & $1.75 \pm 0.18$ & $3.26 \pm 0.11$ & $3.9(7)$ & $7.73 \pm 0.87$ & 3.9 & $1.18 \pm 0.15$ & 8.6 & 170 & $4.27 \pm 0.74$ & 12 \\
\hline $08 / 2003$ & $1.84 \pm 0.18$ & $3.36 \pm 0.09$ & $7.4(7)$ & $7.78 \pm 0.82$ & 3.9 & $1.34 \pm 0.16$ & 9.7 & 170 & $5.13 \pm 0.80$ & 15 \\
\hline $09 / 2003$ & $2.40 \pm 0.41$ & $3.42 \pm 0.15$ & $2.9(6)$ & $9.91 \pm 1.79$ & 5.0 & $1.82 \pm 0.38$ & 13 & 160 & $8.35 \pm 2.27$ & 22 \\
\hline
\end{tabular}

where $I_{0}$ is the differential flux normalization, $E_{0}$ is the energy at which the flux is normalized $(1 \mathrm{TeV})$, and $\Gamma$ is the spectral index of the source. The dashed lines in Fig. 4 show the best $\chi^{2}$ fit to the data of Eq. (3) for the corresponding spectrum. Table 4 shows the best fit results, integral flux (above several thresholds) determined from the fit parameters, and comparisons of these integral fluxes to those measured by H.E.S.S. from the Crab Nebula. Due to the steeper spectral index determined for PKS 2155-304 $\left(\Gamma_{\text {crab }}=2.63 \pm 0.05\right)$, the percentage of the respective Crab fluxes in an individual darkness period is larger for lower thresholds. Although the level of VHE emission from AGN is known to be variable, the integral flux above $300 \mathrm{GeV}$ measured for each of the observation periods shows reasonable agreement with the result reported by Chadwick et al. (1999a), $I(>300 \mathrm{GeV})=\left(4.2 \pm 0.75_{\text {stat }} \pm 2.0_{\text {syst }}\right) \times 10^{-7} \mathrm{~m}^{-2} \mathrm{~s}^{-1}$. In addition to the method described above, an alternative forwardfolding method, used by CAT (Piron et al. 2001), was also applied to quantify the individual spectra and yields very similar results to those shown in Table 4.

\subsection{Time-averaged spectrum}

A time-averaged energy spectrum from all the stereo (2003) data is shown in Fig. 5. The mono-telescope data set is not included for two reasons: the average flux level in 2002 is considerably higher than the rest of the data set. More importantly, the energy is more poorly determined for the monotelescope data set and thus the determination of the spectrum is more susceptible to systematic errors. A fit of the stereo data set to a power law yields $\Gamma=3.32 \pm 0.06, I_{0}=(1.96 \pm 0.12) \times$ $10^{-8} \mathrm{~m}^{-2} \mathrm{~s}^{-1} \mathrm{TeV}^{-1}$, and a $\chi^{2}$ of 10.8 for 7 degrees of freedom. Only the statistical errors are presented. Although the $\chi^{2}$ for the power law fits to the spectra for individual dark periods is reasonable, the $\chi^{2}$ for the time-averaged spectrum corresponds to a probability of 0.148 . In addition, the power law fit to the time-averaged spectrum is above the $90 \%$ upper limit on the differential flux at $3 \mathrm{TeV}$ (not included in the fit). Since this information indicates that a power law is not a good characterization of the data, the energy spectrum is fit to two alternative hypotheses. The first is a power law with an exponential cutoff:

$\frac{\mathrm{d} N}{\mathrm{~d} E}=I_{0}\left(\frac{E}{E_{0}}\right)^{-\Gamma} \mathrm{e}^{-\frac{E}{E_{\text {cut }}}}$,

where $E_{\text {cut }}$ is the cutoff energy, and $I_{0}, E_{0}$, and $\Gamma$ are as defined above. The fit yields an improved $\chi^{2}$ of 6.2 for 6 degrees of freedom (probability $=0.40$ ), and the fit curve falls below all upper limits at higher energies. The best fit values are: $\Gamma=2.90_{-0.23}^{+0.21}, I_{0}=\left(4.0_{-1.2}^{+1.9}\right) \times 10^{-8} \mathrm{~m}^{-2} \mathrm{~s}^{-1} \mathrm{TeV}^{-1}$, and $E_{\text {cut }}=1.4_{-0.7}^{+0.8} \mathrm{TeV}$. The large errors (only statistical errors are shown) on the fit values are the result of strong correlations between the parameters used to characterize the spectral shape. 


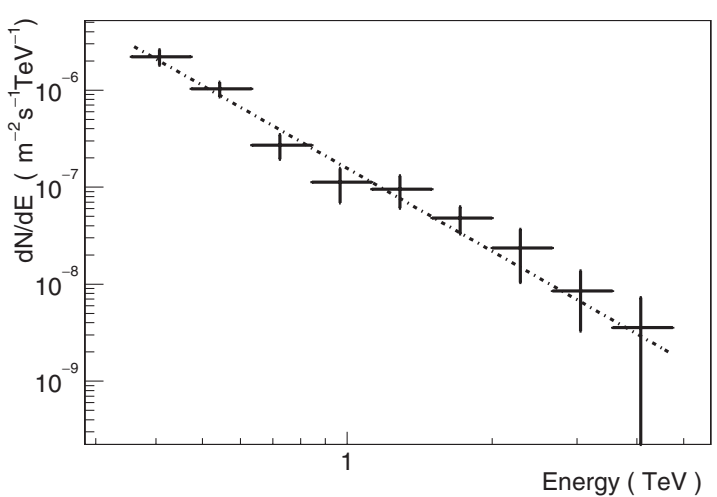

(a)

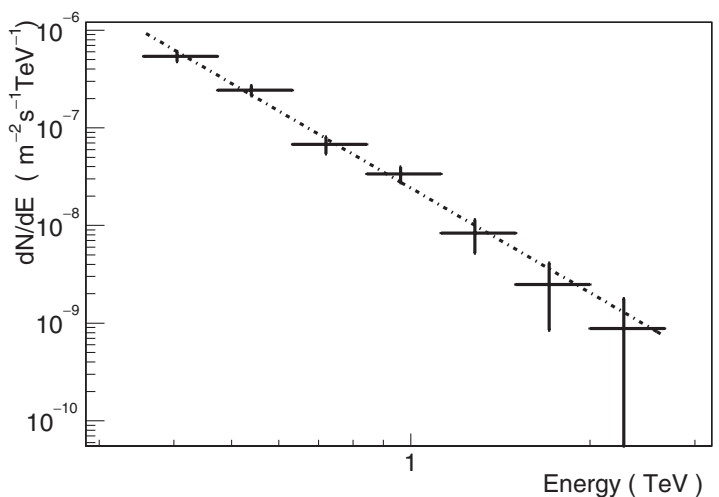

(c)

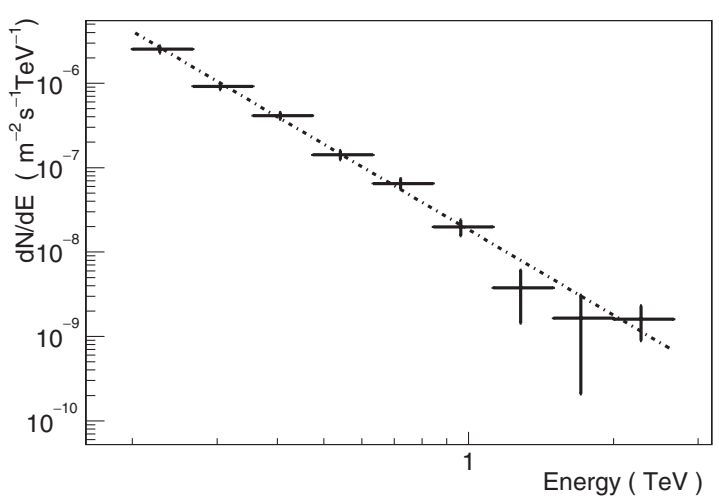

(e)

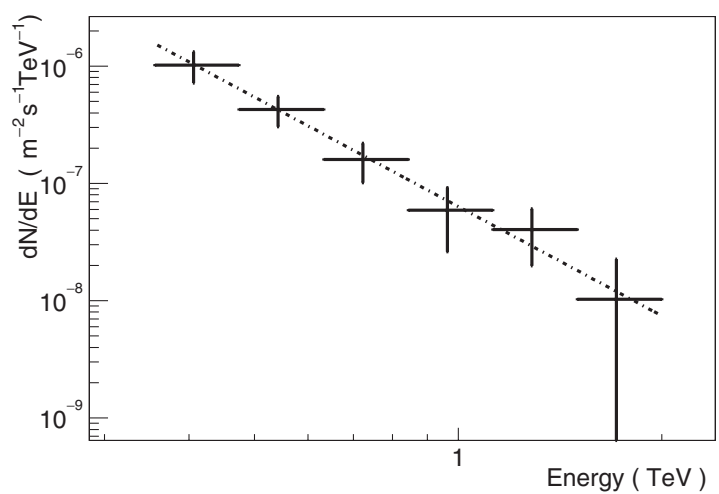

(b)

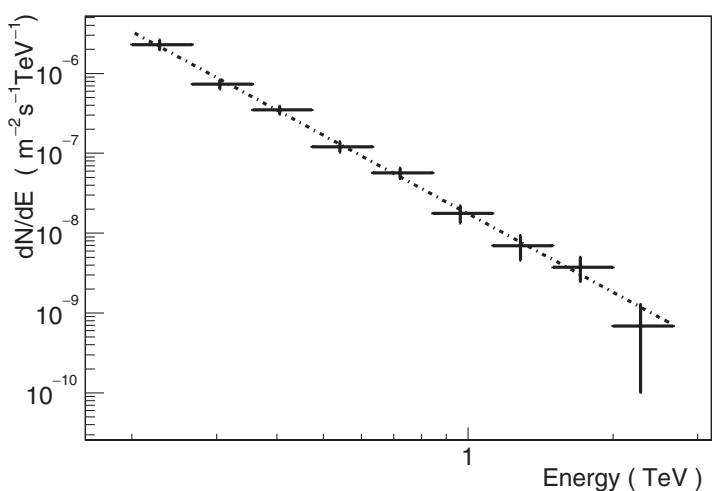

(d)

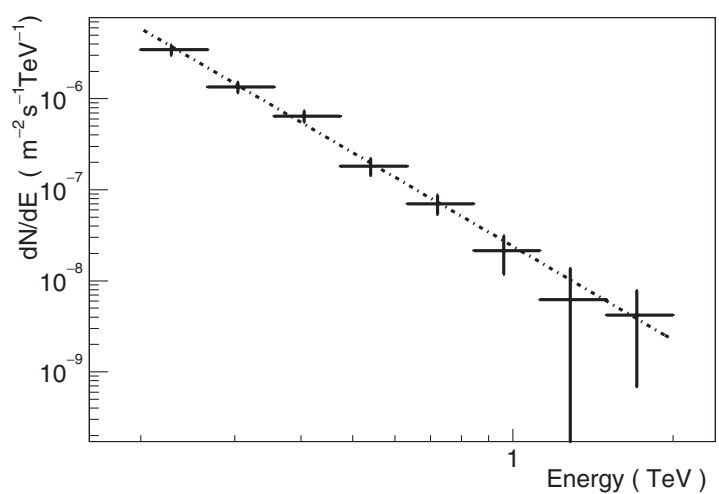

(f)

Fig. 4. The differential energy spectrum measured from PKS 2155-304 for the dark periods of a) July 2002; b) October 2002; c) June 2003; d) July 2003; e) August 2003; f) September 2003. The dashed lines represent the best $\chi^{2}$ fit of the data to a power law.

Although the $\chi^{2}$ resulting from the fit is improved, it cannot be concluded that the fit is significantly better since an F-test shows there is a probability of only 0.92 that the power law with an exponential cutoff is a better characterization of the data.

The second hypothesis is a broken power law, which follows Eq. (3) for energies less than $E_{\text {break }}$, and the following for energies greater than $E_{\text {break }}$ :

$\frac{\mathrm{d} N}{\mathrm{~d} E}=I_{0}\left(\frac{E_{\text {break }}}{E_{0}}\right)^{\left(\Gamma_{2}-\Gamma_{1}\right)}\left(\frac{E}{E_{0}}\right)^{-\Gamma_{2}}$,

where $E_{\text {break }}$ is the energy of the break point in $\mathrm{TeV}, \Gamma_{1}$ and $\Gamma_{2}$ are the spectral indices, and $I_{0}$ and $E_{0}$ are as defined above.
The fit yields a $\chi^{2}$ of 5.1 for 5 degrees of freedom (probability $=0.40$ ), and the fit curve does not fall below the upper limit at $3 \mathrm{TeV}$. The best fit values are: $\Gamma_{1}=3.15_{-0.12}^{+0.10}$, $I_{0}=\left(2.4_{-0.3}^{+0.4}\right) \times 10^{-8} \mathrm{~m}^{-2} \mathrm{~s}^{-1} \mathrm{TeV}^{-1}, \Gamma_{2}=3.79_{-0.27}^{+0.46}$, and $E_{\text {break }}=0.7 \pm 0.2 \mathrm{TeV}$. Only the statistical errors are shown. An F-test shows there is a probability of 0.85 that the broken power law is a better characterization of the data than the simple power law and again it cannot be concluded that the fit is significantly better.

The possible presence of features, such as an exponential cutoff or break, in the energy spectrum could be the result of absorption of $\mathrm{TeV}$ gamma rays by the extragalactic infrared background. However, it may also be intrinsic to the source 

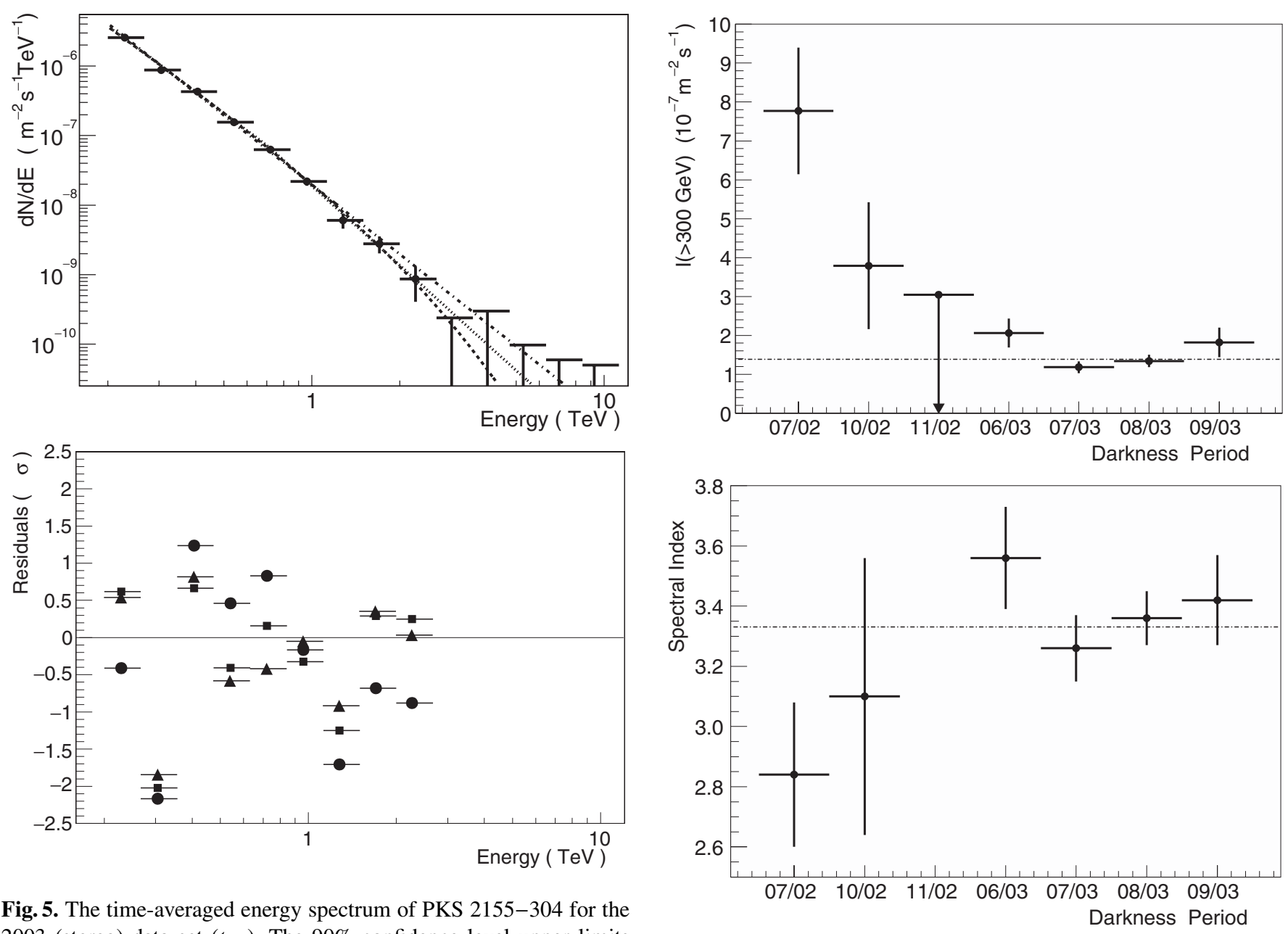

Fig. 5. The time-averaged energy spectrum of PKS 2155-304 for the 2003 (stereo) data set (top). The 90\% confidence level upper limits are determined by the method of Helene (1983) and are not used in the fits. The lines represent the best $\chi^{2}$ fit to a power law (dot-dash), a power law with an exponential cutoff (dashed), and a broken power law (dotted). The bottom figure shows the residuals, (measurement fit) divided by the statistical error, of the best fits to a power law (circles), power law with exponential cutoff (squares), and broken power law (triangles).

spectrum, or the result of absorption of photons close to the source, or both. The distinction can only be made using a larger sample of TeV-emitting AGN at different redshifts, or perhaps by detailed modelling of the broad-band spectrum using data gathered simultaneously at other wavelengths.

\section{Flux variability}

\subsection{Monthly time scale}

Figure 6 shows the integral flux greater than $300 \mathrm{GeV}$ (top) and fit spectral index (bottom) of PKS 2155-304 for each dark period. The dashed line in each plot represents the results from a $\chi^{2}$ fit of the data to a constant. The fit of $I(>300 \mathrm{GeV})$ versus observation period yields a $\chi^{2}$ of 24.0 for 5 degrees of freedom, corresponding to a $2 \times 10^{-4} \chi^{2}$ probability. The data are clearly inconsistent with a constant flux. Although only the statistical errors are used in the fit, the conclusion is unchanged even if systematic errors ( $\sim 20 \%$ for the integral flux) are

Fig. 6. The integral flux above $300 \mathrm{GeV}$ and spectral index versus dark period determined from H.E.S.S. observations of PKS 2155-304. The dashed line represents the best $\chi^{2}$ fit of the data points to a constant. Only the statistical errors are shown.

included. Since PKS 2155-304 was not detected in November 2002 , a $99 \%$ upper limit of $I(>300 \mathrm{GeV})=3.0 \times 10^{-7} \mathrm{~m}^{-2} \mathrm{~s}^{-1}$ was determined by the method of Helene (1983) assuming the 2003 time-averaged spectral index, $\Gamma=3.32$. The upper limit, shown in Fig. 6, is below the flux values from earlier in 2002, but higher than the average flux for the entire data set.

The fit of the spectral index versus period to a constant yields a $\chi^{2}$ of 7.1 for 5 degrees of freedom, corresponding to a $0.21 \chi^{2}$ probability, which is marginally consistent with being constant. Since the fit data points include only the statistical errors, the $\chi^{2}$ will be smaller with the inclusion of systematic errors $(\sim 0.1$ for the spectral index). Therefore, there is no evidence that the spectral index of PKS 2155-304 varies with time. However, the evidence also does not rule out possible temporal variability in the spectral index during the aforementioned large changes in flux.

A correlation between harder spectra and higher flux values might be expected for PKS 2155-304, since this trend was demonstrated for Mkn 421 (Aharonian et al. 2002b) and Mkn 501 (Djannati-Atai et al. 1999; Aharonian et al. 2001). While Fig. 6 is suggestive of such a correlation, the inference is 


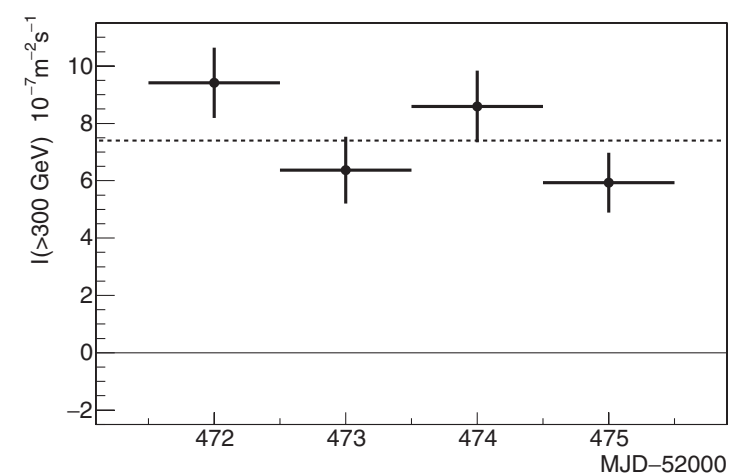

(a)

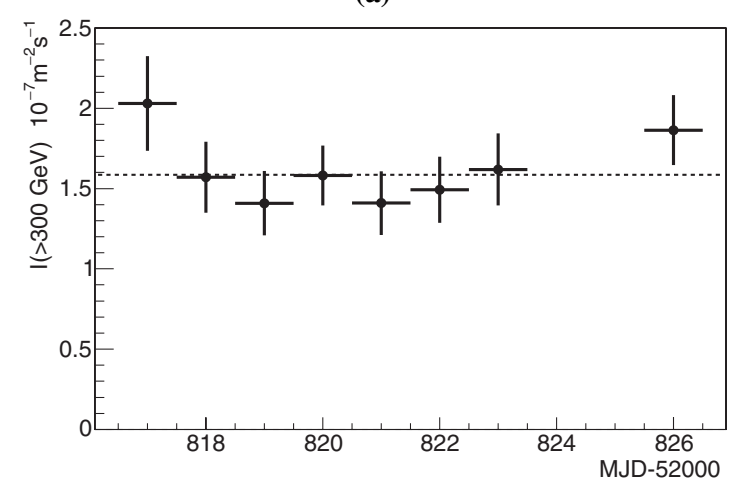

(c)

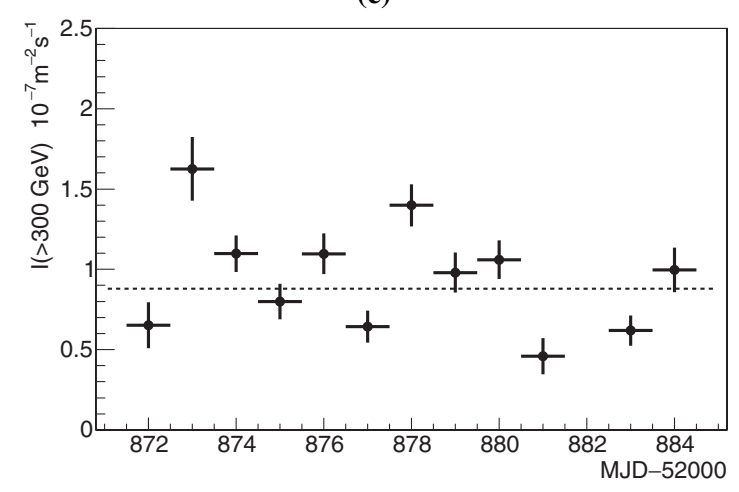

(e)

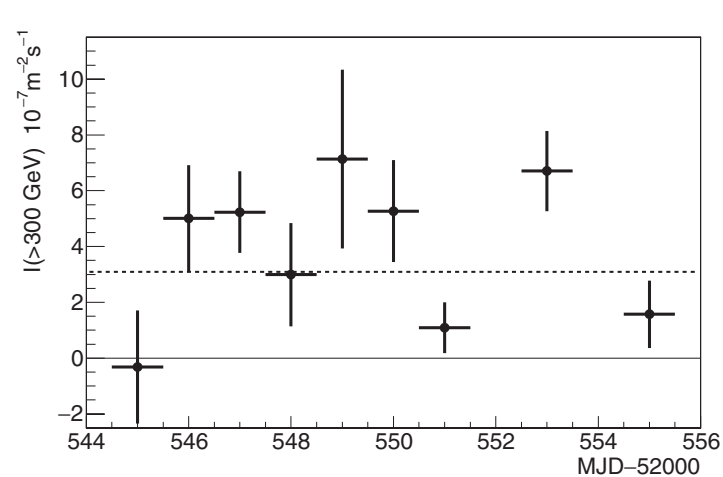

(b)

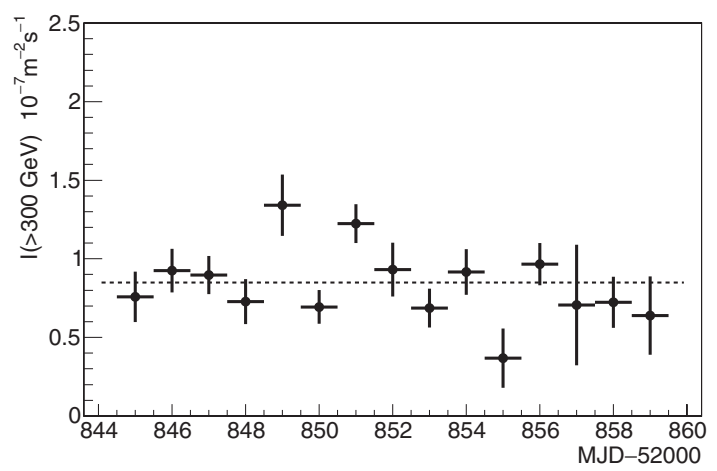

(d)

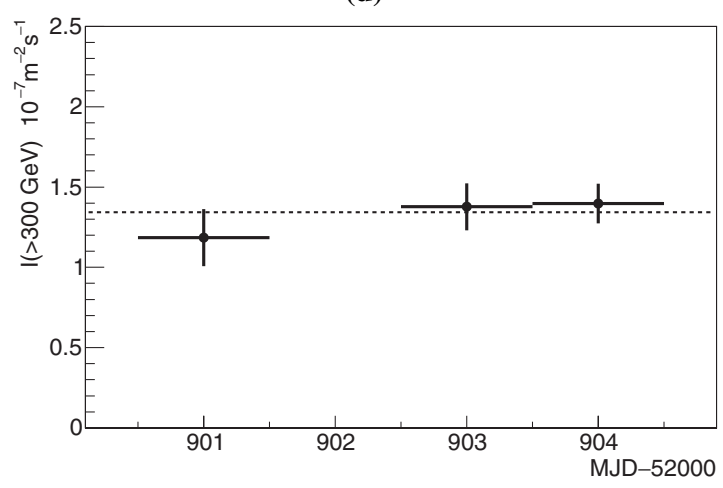

(f)

Fig. 7. The nightly integral flux greater than $300 \mathrm{GeV}$ versus time measured from PKS 2155-304 in a) July 2002; b) October 2002; c) June 2003; d) July 2003; e) August 2003; f) September 2003. Only the statistical errors are shown. The dashed lines represent the best $\chi^{2}$ fit of the data to a constant for each darkness period.

mainly drawn from the July 2002 data point, taken during the first month of H.E.S.S. operations where only one telescope was operational, and thus the fit spectrum is most likely to have larger systematic errors. In addition, the spectral index was shown to be marginally consistent with being constant. Therefore, further observations with the more sensitive complete H.E.S.S. array are needed to make a definitive statement regarding any correlation.

Given the large significance observed in each month of data taking, a variability search on a monthly time scale represents a rather coarse temporal binning of the data. Therefore studies were performed on the data to determine if the VHE flux from PKS 2155-304 is also variable on shorter time scales.

\subsection{Nightly time scale}

The integral flux greater than $300 \mathrm{GeV}$ measured from PKS 2155-304 is calculated for each night of observations and is shown in Fig. 7. The exposure is different in the various nights and the spectral index assumed in the flux calculation is the value determined from the power law fit to the timeaveraged spectra for $2003(\Gamma=3.32)$. This value is used since there is no strong evidence for variability in the spectral index. Table 5 lists the results of the fit of the nightly fluxes during each month to a constant. The poor $\chi^{2}$ values for some of the dark periods show that variability in the flux of VHE gammarays from PKS 2155-304 clearly occurs on time scales of days. However, the June and September 2003 results also show that there are periods of steady flux as well. 
Table 5. The $\chi^{2}$, degrees of freedom (d.o.f.), and $\chi^{2}$ probability, from the best fits to a constant of the nightly integral flux greater than $300 \mathrm{GeV}$ observed from PKS 2155-304 versus time for each dark period.

\begin{tabular}{rccc}
\hline \hline Dark period & $\chi^{2}$ & d.o.f. & $P\left(>\chi^{2}\right)$ \\
\hline $07 / 2002$ & 6.4 & 3 & $9.5 \times 10^{-2}$ \\
$10 / 2002$ & 21.8 & 8 & $5.4 \times 10^{-3}$ \\
$06 / 2003$ & 5.7 & 7 & 0.58 \\
$07 / 2003$ & 30.1 & 14 & $7.4 \times 10^{-3}$ \\
$08 / 2003$ & 74.9 & 12 & $3.7 \times 10^{-11}$ \\
$09 / 2003$ & 1.0 & 2 & 0.60 \\
\hline
\end{tabular}

$z$

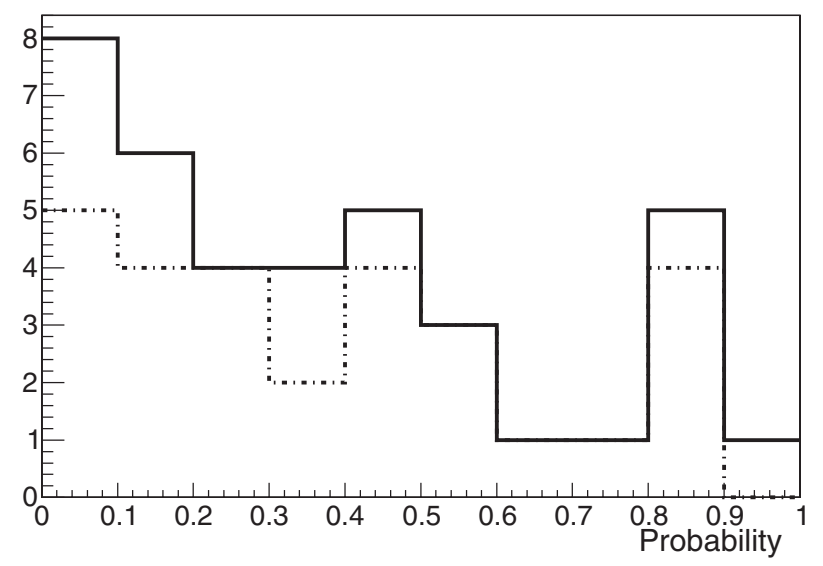

(a)

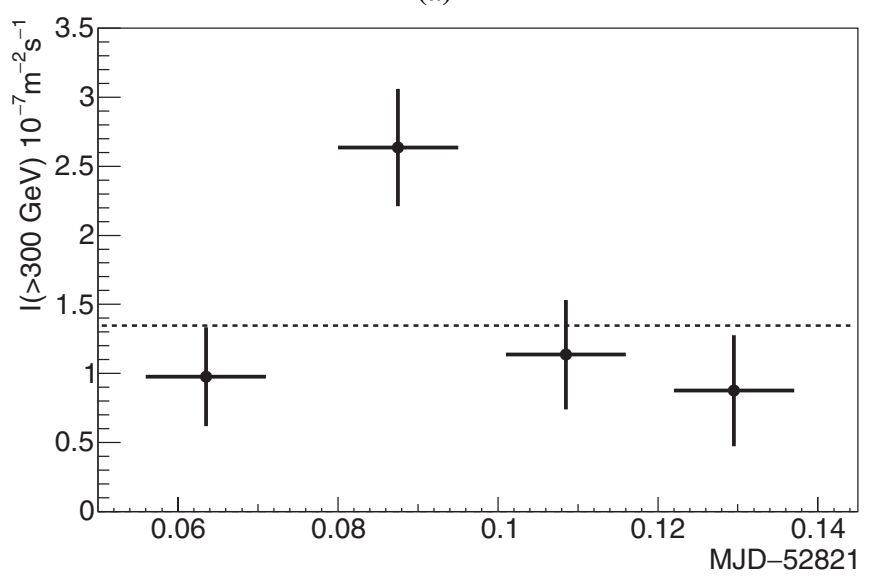

(b)

Fig. 8. a) The distribution of $\chi^{2}$ probabilities from best fits to a constant of the run by run integral flux greater than $300 \mathrm{GeV}$ for each night of H.E.S.S. observations of PKS2155-304 in 2003. The solid line is for all nights of data taking and the dashed line represents nights with more than one degree of freedom (3 runs minimum). b) The run by run integral flux greater than $300 \mathrm{GeV}$ observed from PKS 2155-304 for the night of MJD 52821. Only the statistical errors are shown.

\subsection{Sub-hour time scale}

A search for evidence of intra-night variability in the VHE flux from PKS 2155-304 is performed for the 2003 data set. A run by run ( $28 \mathrm{~min}$ ) time scale is chosen for simplicity and because each stereo run should yield on average $\sim 4 \sigma$ given that $\sim 44 \sigma$ was observed in 55.4 live time hours of stereo observation.
The study is not performed for the 2002 data because the monoconfiguration is considerably less sensitive and the data are more sparse (one on-source run on most nights). The respective fluxes greater than $300 \mathrm{GeV}$ for each run are fit to a constant for each night of observation, with the hypothesis that any variability will manifest itself in the form of a poor $\chi^{2}$. Only runs with zenith angles of less than 45 degrees are analyzed to eliminate possible systematic effects from large zenith angle observations. As motivated in the previous section, the assumed spectral index for the flux calculation is $\Gamma=3.32$. Since there are differing numbers of runs in a given night, the $\chi^{2}$ probability is calculated from the fit to a constant for each night and is shown by the solid line of Fig. 8a for all nights with a minimum of one degree of freedom. The dashed line in Fig. 8a represents the nights with more than one degree of freedom. A larger population of nights with low probabilities exists and is evidence for intra-night flux variability.

Figure $8 \mathrm{~b}$ shows the flux light curve for the night with the lowest probability of being consistent with constant along with the corresponding fit (dashed line). On this night, MJD 52821 , flaring behavior is suggested since the flux increases by a factor of $2.7 \pm 0.7$ on the time scale of half an hour, and then decreases by a factor of $2.3 \pm 0.9$ in a similar time. Unfortunately at this stage in the construction of H.E.S.S., observations were only possible with the less sensitive two-telescope software stereo configuration, thus a more detailed study is not possible due to the relatively small number of observed gamma rays ( $\sim 1$ per min). However, with the completed H.E.S.S. experiment it will be possible to explore similar phenomena on a time scale of a few minutes.

\section{Conclusions}

Gamma rays with energies greater than $160 \mathrm{GeV}$ have been detected with high significance $(\sim 45 \sigma)$ from the HBL object PKS 2155-304 in H.E.S.S. observations made in 2002 and 2003. Although observations were made in less sensitive configurations (fewer telescopes) of H.E.S.S. before the completion of the construction of the full experiment, a strong detection is found in each of the dark periods of observations with appreciable exposure. The VHE flux observed from PKS 2155-304 is shown to be variable on the time scale of months, nights, and hours with an average flux above $300 \mathrm{GeV}$ between $10 \%$ and $60 \%$ of that observed from the Crab Nebula. An extreme case of variability shows an increase in the observed flux by a factor of $2.7 \pm 0.7$ in a 30 min interval followed by a decrease by a factor of $2.3 \pm 0.9$ in the following $30 \mathrm{~min}$. Energy spectra are made for each dark period for which a detection occurred and are found to be characterized by a steep power law. No evidence is found for variability in the spectral index of PKS 2155-304 over time, or for a hardening of the spectrum with increased flux levels. However, these hypotheses are also not ruled out. A time-averaged energy spectrum is determined for the 2003 H.E.S.S. observations and fits to a power law with an exponential cutoff $\left(\Gamma=2.90_{-0.23}^{+0.21}\right.$, $\left.E_{\text {cut }}=1.4_{-0.7}^{+0.8} \mathrm{TeV}\right)$, and a broken power law $\left(\Gamma_{1}=3.15_{-0.12}^{+0.10}\right.$, $\left.\Gamma_{2}=3.79_{-0.27}^{+0.46}, E_{\text {break }}=0.7 \pm 0.2 \mathrm{TeV}\right)$, show an improved $\chi^{2}$ per degree of freedom relative to the one obtained from a fit 
to a simple power-law $(\Gamma=3.32 \pm 0.05$ ). Although the fits to power laws with features are not significantly better than that of a fit to a simple power law, the suggestion of features may indicate the absorption of $\mathrm{TeV}$ photons on the extragalactic infrared background light. However, any spectral curvature could also be intrinsic to the blazar.

Comparison of the properties of PKS 2155-304 measured by H.E.S.S. to those of other blazars detected at $\mathrm{TeV}$ energies is somewhat difficult due to the highly variable nature of these objects and their differing redshifts. Regardless, comparison of the flux (magnitude and variability) and spectra, may yield some insights into phenomena associated with $\mathrm{TeV}$ bright AGN. In the cases of the most well studied TeV blazars, Mkn $421(z=0.030)$ and Mkn $501(z=0.033)$, the observed flux has been found to vary from a fraction of the Crab flux to peak values of $\sim 7$ (Aharonian et al. 2002b) and $\sim 10$ (Aharonian et al. 1999a) times the Crab flux respectively. The flux detected from 1ES $1959+650(z=0.047)$ ranges from 5\% to $220 \%$ of the Crab (Aharonian et al. 2003b), and the flux measured from 1 ES $1426+428$ has shown variability by a factor of 2.5 , with peak fluxes of order $10 \%$ of the Crab (Aharonian et al. 2003a). Clearly, the flux observed from PKS 2155-304, which ranges from $10 \%$ to $60 \%$ of the Crab, does not vary as much as that from Mkn 421, Mkn 501 and 1ES 1959+650. Additionally, the peak fluxes observed from these objects are considerably higher than that measured for PKS 2155-304. The observed flux from PKS 2155-304 is most similar, both in magnitude and variability, to that found from 1ES $1426+428$. Comparing the spectra of the other AGN to that found from PKS 2155-304 also shows some differences. The spectral indices, prior to the exponential cutoffs found, of Mkn $501(\Gamma=1.92 \pm 0.20), \operatorname{Mkn} 421(\Gamma=2.19 \pm$ $0.04)$, and $1 \mathrm{ES} 1959+650(\Gamma=1.83 \pm 0.17)$ are harder than $\Gamma=3.32 \pm 0.05$ found for PKS 2155-304. Again the most similar blazar is $1 \mathrm{ES} 1426+428$, whose spectral index is $\Gamma=$ $3.50 \pm 0.35$ (Petry et al. 2002). It is interesting that the properties of PKS 2155-304 $(z=0.117)$ are most like those of 1ES $1426+428$, whose redshift $(z=0.129)$ is also the most similar. However, it cannot be stated from the evidence presented that the similarities are a direct consequence of the comparable redshifts.

As of 2004, the H.E.S.S. detector is complete and is considerably more sensitive than any of the configurations presented here, allowing for more detailed studies of PKS 2155-304 and other astrophysical objects to be performed in the future.

Acknowledgements. The support of the Namibian authorities and of the University of Namibia in facilitating the construction and operation of H.E.S.S. is gratefully acknowledged, as is the support by the German Ministry for Education and Research (BMBF), the MaxPlanck-Society, the French Ministry for Research, the CNRS-IN2P3 and the Astroparticle Interdisciplinary Programme of the CNRS, the UK. Particle Physics and Astronomy Research Council (PPARC), the IPNP of the Charles University, the South African Department of Science and Technology and National Research Foundation, and by the University of Namibia. We appreciate the excellent work of the technical support staff in Berlin, Durham, Hamburg, Heidelberg, Palaiseau, Paris, Saclay, and in Namibia in the construction and operation of the equipment.

\section{References}

Aharonian, F. A., Akhperjanian, A. G., Barrio, J. A., et al. 1999a, A\&A, 342, 69

Aharonian, F. A., Akhperjanian, A. G., Barrio, J. A., et al. 1999b, A\&A, 349, 11

Aharonian, F., Akhperjanian, A., Barrio, J., et al. 2001, ApJ, 546, 898

Aharonian, F., Akhperjanian, A., Barrio, J., et al. 2002a, A\&A, 384, L23

Aharonian, F., Akhperjanian, A., Beilicke, M., et al. 2002b, A\&A, 393, 89

Aharonian, F., Akhperjanian, A., Beilicke, M., et al. 2003a, A\&A, 403,523

Aharonian, F., Akhperjanian, A., Beilicke, M., et al. 2003b, A\&A, 406, L9

Aharonian, F., Akhperjanian, A. G., Aye, K.-M., et al. 2004, Astroparticle Physics, 22, 109

Brinkmann, W., Maraschi, L., Treves, A., et al. 1994, A\&A, 288, 443

Bernlöhr, K., Carrol, O., Cornils, R., et al. 2003, Astroparticle Physics, 20,111

Catanese, M., et al. 1995, Proc. of the Workshop Towards a Major Cherenkov Detector (Padova), 335

Cooke, B. A., Ricketts, M. J., Maccacaro, T., et al. 1978, MNRAS, 182, 489

Chadwick, P. M., Lyons, K., McComb, T. J. L., et al. 1999, ApJ, 513, 161

Chadwick, et al. 1999, Proc. of the 26th ICRC (Salt Lake City), OG 2.1.13.

Chiappetti, L., \& Torroni, V. 1997, IAU Circ., 6776

Davies, J., \& Cotton, E. 1957, J. Solar Energy, 1, 16

Djannati-Atai, A., Piron, F., Barrau, A., et al. 1999, A\&A, 350, 17

Funk, S., Hermann, G., Hinton, J., et al. 2004, Astropart. Phys., 22(3-4), 285

Gillessen, S. 2003, Proc. of the 28th ICRC (Tsukuba), 2899

Griffiths, R. E., Briel, U., Chaisson, L., Tapia, S., et al. 1979, ApJ, 234, 810

Helene, O. 1983, Nuclear Instruments \& Methods, 212, 319

Hillas, A. 1985, Proc. of the 19th ICRC (La Jolla), 3, 445

Hofmann, W. 2003, Proc. of the 28th ICRC (Tsukuba), 2811

Li, T., \& Ma, Y. 1983, ApJ, 272, 317

Masterson, C. P. 2000, Ph.D. Thesis, National University of Ireland

Nakase, et al. 2003, Proc. of the 28th ICRC (Tsukuba), 2587

Nicastro, F., Zezas, A., Drake, J., et al. 2002, ApJ, 573, 157

Nishijima, et al. 2001, Proc. of the 27th ICRC (Hamburg), 2626

Mohanty, G., Biller, S., Carter-Lewis, D. A., et al. 1998, Astropart. Phys., 9, 15

Petry, D., Bond, I. H., Bradbury, S. M., et al. 2002, ApJ, 580, 104

Piron, F., Djannati-Atai, A., Punch, M., et al. 2001, A\&A, 374, 895

Roberts, M. D., McGee, P., Dazeley, S. A., et al. 1999, A\&A, 343, 691

Schwartz, D. A., Griffiths, R. E., Schwarz, J., et al. 1979, ApJ, 229, L53

Vestrand, W. T., Stacy, J. G. \& Sreekumar, P. 1995, ApJ, 454, L93

Vestrand, W. T., \& Sreekumar, P. 1999, Astropart. Phys., 11, 197

Vincent, W. T., Stacy, J. G., Sreekumar, P., et al. 2003, Proc. of the 28th ICRC (Tsukuba), 2887

Wandel, A., \& Urry, C. M. 1991, ApJ, 367, 78 